\title{
20 Jahre im Zentralvorstand - eine Bilanz
}

\section{Yves Guisan}

Ehemaliger Vizepräsident der FMH, ehemaliger Nationalrat

20 Jahre Standespolitik, 14 Jahre Politik: Welche Bilanz lässt sich daraus ziehen? Die Bilanz fällt zwiespältig aus. Genauer gesagt ist sie eine Mischung aus der Befriedigung, die mit solchen Funktionen zweifellos verbunden ist, und aus der Erfahrung, dass im Gesundheitsbereich ein permanenter, unaufhörlicher und mühsamer Kampf auszufechten ist. Im Rahmen dieser Auseinandersetzungen bestehen Erfolge viel häufiger im Aufschub von Massnahmen als in der Entwicklung wirklich neuer Denkansätze. Die jüngsten Aussichten, die stark von den Abstimmungsergebnissen abhängen, bieten jedoch die Voraussetzungen für einige positive Veränderungen.

\section{Jahre Standespolitik, 14 Jahre Politik: Welche Bilanz lässt sich daraus ziehen?}

Korrespondenz:

Dr. med. Yves Guisan Le Moulin

CH-1660 L'Etivaz

yvesguisan@sapphirenet.gi
Meine Tätigkeit bei der FMH wurde sofort durch die Probleme mit dem damaligen Kranken- und Unfallversicherungsgesetz KUVG, anschliessend durch die Übergangslösung des Kranken- und Mutterschaftsversicherungsgesetzes und schliesslich durch das Bundesgesetz über die Krankenversicherung KVG geprägt. Ausserdem hatte ich es mit drei verschiedenen Präsidenten zu tun, und ich war mit einer bedeutenden Änderung der Überlegungen und Anliegen konfrontiert, insbesondere was die Rolle des Arztes im Gesundheitssystem anbelangt.

In den achtziger Jahren führte der massive Missbrauch der Risikoselektion zu einer tiefgreifenden strukturellen Krise im Bereich des KUVG. Da die Prämien im Rahmen der nichtobligatorischen Versicherung abhängig vom Alter und von den Risiken festgelegt werden, kämpften die Krankenkassen noch härter um junge und gesunde Versicherte. Vor diesem Hintergrund war es für ältere Menschen nicht mehr möglich, die Krankenkasse zu wechseln, und sie wurden mit exorbitanten Prämien konfrontiert. Daraus resultierte auch eine immer höhere Belastung für den Bund und die Kantone, die pro Kind, Frau und Mann individuelle Beiträge entrichteten, welche zusätzlich entsprechend den Gesundheitskosten indexiert wurden.
Verschiedene Massnahmen wurden ergriffen, um diese Krise zu bewältigen, die man durchaus als existenziell qualifizieren konnte. Zunächst beschloss der Bund, seinen Beitrag auf zwei Milliarden zu beschränken und seine Beiträge nicht mehr gemäss den Kosten zu indexieren. Anschliessend realisierte er ein Notprogramm im Rahmen des Kranken- und Mutterschaftsversicherungsgesetzes, mit dem eine ganze Reihe von drastischen Massnahmen eingeführt wurde: Wahlfranchise mit einer maximalen Beteiligung von 750 Franken pro Fall, Selbstbehalt für ambulante Leistungen von 20\% bei Erwachsenen und $10 \%$ bei Kindern, Beteiligung der Patientinnen und Patienten an den Unterkunfts- und Behandlungskosten im Spitalbereich und schliesslich obligatorischer Vertrauensarzt für jede Krankenkasse, was bis dahin nicht der Fall gewesen war. Die Regelung bei der Entschädigung der Ärztinnen und Ärzte und der Fachpersonen in den paramedizinischen Berufen im allgemeinen wurde verschärft, indem für die ganze Schweiz eine einheitliche Methodik eingeführt werden sollte, für die der Bundesrat gegebenenfalls die Grundsätze festlegen würde. Kurz gesagt sollte alles viel strenger kontrolliert werden. Glücklicherweise wurde das im März 1987 vom Parlament verabschiedete Kranken- und Mutterschaftsversicherungsgesetz in der Referendumsabstimmung vom 6. Dezember 1987 abgelehnt. Doch es hatte zweifellos gewisse Meilensteine für die künftige Entwicklung gesetzt. In der darauffolgenden Woche wurde ich zusammen mit Max Giger in den Zentralvorstand gewählt.

Wir erlebten auf Anhieb eine sehr stürmische Zeit. Mit der sogenannten «Krankenkasseninitiative» wurde versucht, eine andere Lösung für die Kontrolle und Eindämmung der Kosten zu unterbreiten. Doch die Vorlage war sehr allgemein formuliert. Sie wurde von den Stimmbürgerinnen und Stimmbürgern ebenfalls verworfen. Anschliessend beauftragte der Bundesrat vier Experten mit der Erarbeitung einiger konkreter Vorschläge. Parallel dazu wurden dringliche Bundesbeschlüsse erlassen, um die Tarife, Beiträge und Prämien einzufrieren.

Der Zentralvorstand der FMH agierte damals sehr diszipliniert, sehr höflich und sehr förmlich. Der Präsident, Dr. Hans-Ruedi Sahli, war ein Mann alter Schule und genoss bei allen grossen 
Respekt. Im Zusammenhang mit der Revision des KUVG war unser Hauptanliegen die Erhaltung unserer Unabhängigkeit gegenüber den Krankenkassen und die Gewährleistung der therapeutischen Freiheit. Selbstverständlich bestand die Möglichkeit, dass sich die Versicherungsprobleme auf die finanzielle Beziehung mit dem Versicherten auswirkten. Doch wir wollten unsere Unabhängigkeit und die Möglichkeit bewahren, mit den Versicherern auf einer möglichst wenig regulierten Basis Rahmenvereinbarungen abzuschliessen. Was die therapeutische Freiheit anbelangt, handelte es sich um ein sakrosanktes Prinzip, das wir nicht preisgeben wollten. Dies ist auch heutzutage nach wie vor der Fall. Wir hatten eine sehr individualistische Vorstellung von Medizin, die auf die Beziehung zwischen Arzt und Patient beschränkt war und nicht in ein Gesundheitssystem oder in einen allgemeineren gesellschaftlichen Zusammenhang integriert werden sollte. Diese Auffassungen vertraten Präsident Sahli und unser damaliger Generalsekretär François-Xavier Deschenaux im Rahmen der ausserparlamentarischen Kommission Schoch (Otto Schoch war damals Ständerat von Appenzell Ausserrhoden). Diese Kommission war vom Bundesrat eingesetzt worden, um einen Vorschlag für die Revision des KUVG zu erarbeiten. Dabei sollte sich die Kommission an einigen grundlegenden Prinzipien orientieren, die der Bundesrat vorgängig festgelegt hatte.

Auf diese Weise wurde die FMH eng in die Erarbeitung des Expertenberichts einbezogen, der am 2. November 1990 veröffentlicht wurde. Ein Jahr danach folgte die Botschaft des Bundesrates zum KVG, die am 6. November 1991 publiziert wurde. Wir intervenierten damals während der parlamentarischen Debatten, wobei wir die Regeln der Achtung vor den Parlamentarierinnen und Parlamentariern beachteten, die zum dama-

\section{Das gegenwärtig geltende KVG wurde 1992 vom Volk mit einer knappen Mehrheit von 71115 Stimmen angenommen, was einem Ja-Anteil von 51,8\% entsprach}

ligen Zeitpunkt noch galten. Das KVG wurde schliesslich ohne Begeisterung gutgeheissen, und sogar die FMH gab ein entsprechendes Lippenbekenntnis ab. Da die Meinungen innerhalb der FMH sehr geteilt waren, verzichteten wir im $\mathrm{Zu}$ sammenhang mit der Abstimmung vom 4. Dezember 1994 auf eine Kampagne. Das gegenwärtig geltende Gesetz wurde vom Volk mit
1021175 gegen 950060 Stimmen und damit mit einer knappen Mehrheit von 71115 Stimmen angenommen, was einem Ja-Anteil von 51,8\% entsprach.

Die FMH befasste sich in der Folge mit einem Parameter, der für uns von ausschlaggebender Bedeutung war: mit der praktischen Umsetzung von Art. 43 Abs. 5, in dem folgendes festgelegt ist: «Einzelleistungstarife müssen auf einer gesamtschweizerisch vereinbarten einheitlichen Tarifstruktur beruhen. Können sich die Tarifpartner nicht einigen, so legt der Bundesrat diese Tarifstruktur fest.» Diese Arbeiten wurden anschliessend im Zusammenhang mit der damals laufenden Unfalltarifrevision fortgesetzt, die unter dem Kürzel GRAT (Gesamt-Revision des ArztTarifes) durchgeführt wurde. Diese Revision war im Herbst 1987 im Rahmen einer gemeinsamen Vereinbarung und in einem verhältnismässig guten Einverständnis mit der MTK beschlossen worden. Ich war seit meiner Wahl in den Zentralvorstand der FMH im Jahr 1987, d.h. seit dem Beginn der Grundsatzverhandlungen, Mitglied dieser Verhandlungsdelegation. Es ging darum, eine konkrete Grundlage für den Begriff «Betriebswirtschaft» zu erarbeiten. Diese sollte die Wiederherstellung des Gleichgewichts zwischen den verschiedenen Fachrichtungen ermöglichen und die Voraussetzungen dafür schaffen, dass künftig neue Leistungen ohne Probleme berechnet werden könnten. Ausserdem ging es darum, den gesetzlichen Rahmen für die Arztpraxen festzulegen. Die entsprechenden Arbeiten erlaubten nicht ohne Schwierigkeiten im Jahr 1994, die Grundregeln des neuen Tarifs festzulegen. Zum damaligen Zeitpunkt schlossen sich die Krankenkassen dieser Arbeitsgruppe an. Dies erfolgte unter der Voraussetzung, dass der damalige Stand der Arbeiten übernommen wurde, ohne ihn in Frage zu stellen. Dies war jedoch leider nicht der Fall. Deshalb wurde die Aufgabe durch die Mitarbeit des «Konkordats» und anschliessend von santésuisse in der Verhandlungsgruppe erheblich erschwert. Ich will mich an dieser Stelle nicht lang und breit über alle Einzelheiten dieser langwierigen Verhandlungen auslassen. Zusammenfassend kann jedoch festgehalten werden, dass sich die Verhandlungen rasch von den festgelegten Grundsätzen entfernten und sich in eine praktisch ausschliesslich technische Richtung entwickelten. Und ganz ehrlich gesagt wurde bald einmal wie auf einem Basar verhandelt ...

Im Jahr 1994 erfolgte auch im Präsidium ein Wechsel. Da der neue Präsident, Hans Heinrich Brunner, mehr ökonomisch und technisch ausgerichtet war, kam ihm diese neue Herausforde- 
rung gewissermassen wie gerufen. Er machte sie $\mathrm{zu}$ «seiner Angelegenheit», so dass der Zentralvorstand fortschreitend von der gesamten Kontrolle der Geschäfte quasi ausgeschlossen wurde. Die praktisch obligatorische Mitgliedschaft in der FMH wurde kurz- oder mittelfristig durch die Einführung von staatlichen Weiterbildungsdiplomen gefährdet. Diese mussten definitionsgemäss für alle und damit auch für Nichtmitglieder zugänglich sein. Der Nutzen der Mitgliedschaft war somit mit konkreten Vorteilen zu rechtfertigen, die über die Berufsbildung und die Gewerkschaftstätigkeit hinausgingen. Es galt, die Attraktivität der Standesorganisation beträchtlich zu erhöhen. Dies musste in erster Linie auf wirtschaftlichen Vorteilen und nur nebenbei auf Fragen beruhen, die mit den Standesinteressen zusammenhingen. Es ging also um die Aufwertung von FMH Services, die Schaffung des HIN und anschliessend von Bluecare, um die Unterstützung von AESA, einem Genfer Vertriebsunternehmen für medizinisches Material, sowie um die Folgearbeiten im Zusammenhang mit den Auswirkungen des neuen TARMED-Tarifs auf der Ebene der Datenverarbeitung. Dabei handelt es sich nicht um eine abschliessende Aufzählung.

Diese Massnahmen brachten offensichtlich keinen Erfolg und führten im Zusammenhang mit Eigenmächtigkeit und Desinformation zu extremen finanziellen Spannungen. Sie haben im Verlauf dieser Legislatur die entsprechenden Sanierungsmassnahmen in die Wege geleitet. Doch insbesondere dieser technischen und wirtschaftlichen Vision, die im Rahmen einer vermeintlich sehr kultivierten Debatte entwickelt wurde, fehlte es weitgehend an grundsätzlicheren Konzepten hinsichtlich der Rolle der Ärzteschaft und unseres Standes innerhalb des Gesundheitssystems. Statt solcher Konzepte standen Überlegungen im Vordergrund, bei denen es praktisch ausschliesslich um die Kräfteverhältnisse zwischen den Partnern ging. Aus diesem Grund konnten wir keine Lösungen für grundsätzlichere Probleme und Gefährdungen im Zusammenhang mit der Vertragsfreiheit und dem ausschliesslich wirtschaftlichen Wettbewerb entwickeln.

Durch diesen Mangel an politischen und gesellschaftlichen Visionen innerhalb der FMH wurden exzentrische Tendenzen verstärkt, die zur Bildung von verschiedenen Interessengruppen führten. Es bestehen indessen durchaus andere Instrumente, mit denen sich die Gesundheitskosten kontrollieren lassen, ohne dass dadurch der bedeutende soziale und gesellschaftliche Auftrag des Gesundheitssystems gefährdet wird. Gleichzeitig kann in diesem Rahmen auch unseren Standesinteressen Achtung verschafft werden. In der Folge wurde ich vom Zentralvorstand gemäss den alten Statuten zum Vizepräsidenten der FMH ernannt und anschliessend von Ihnen in diesem Amt bestätigt. Es erwies sich als äusserst schwierig, mit dem Präsidenten die Diskussion über diese wesentlichen Fragen in Gang zu bringen. Ausserdem waren wir im Zentralvorstand nur wenige, die zu diesem Themenbereich die entsprechenden Fragen an den Präsidenten richteten, so dass wir entweder rasch in die Schranken gewiesen oder in eine Minderheitsposition gedrängt wurden.

Die Zeiten haben sich deutlich geändert. Es reicht nicht mehr, als Arzt eine andere Meinung $\mathrm{zu}$ vertreten, um recht zu bekommen. Die einstigen Vorrechte unseres Standes werden heutzutage zu Recht als Arroganz betrachtet. Man muss heute in der Lage sein, Alternativvorschläge zu unterbreiten und überzeugend $\mathrm{zu}$ argumentieren. In diesem Bereich wiesen wir bis vor kurzem zweifellos gewisse Defizite auf.

Als Nationalrat sah ich mich ab 1995 auf mich allein gestellt. Was den Gesundheitsbereich anbelangt, betrachtete ich mich in erster Linie als Vertreter der FMH. Die verschiedenen Vorschläge, die ich Hans Heinrich Brunner unterbreitete, wurden von ihm in der Regel gutgeheissen. Gleichzeitig wurden aber jeweils aus unseren Reihen und von Interessengruppen Äusserungen verbreitet, die in eine völlig andere Richtung gingen. Diese anderslautenden Meinungsäusserungen trugen auf politischer Ebene nicht unbedingt zur Glaubwürdigkeit meiner Vorschläge bei.

\section{Es reicht nicht mehr, als Arzt eine andere Meinung zu vertreten, um recht zu bekommen}

Die Grundsätze des KVG sollen nicht in Frage gestellt werden, doch dieses Gesetz ist auch mit grossen Anwendungsproblemen verbunden. Dies gilt insbesondere für die folgenden Bereiche: Kompetenzverteilung zwischen Bund und Kantonen, Belastung des Mittelstands bis zur Grenze des Erträglichen durch das Solidaritätsprinzip mit sehr ungleichen kantonalen Beitragssystemen und unzureichender Ausgleichsfonds, um die Risikoselektion auszuschliessen und im Hinblick auf Effizienz und Qualität den Wettbewerb zwischen den Versicherern zu gewährleisten. Die Spitalfinanzierung und Spitalplanung wurden 
durch den Entscheid der Eidgenössischen Räte vom letzten Dezember teilweise zufriedenstellend gelöst. Im Zentrum all dieser Anstrengungen steht die Eindämmung der Kosten mit dem nun wieder aufgekommenen Vorschlag zur Einführung der Kontrahierungsfreiheit. Diese verschiedenen Fragen wurden im Rahmen der drei KVGRevisionen, die seit 1999 durchgeführt wurden, immer wieder aufgeworfen.

Bislang sind wir der viel zitierten Vertragsfreiheit entronnen, die immer wieder als Wunderlösung präsentiert wird. Doch mehrmals hat nicht viel zu deren Einführung gefehlt. Ein erster Versuch erfolgte über eine parlamentarische Initiative von Nationalrat Hansueli Raggenbass, CVP (TG), Direktor der Sanitas. Dieser Versuch, der parallel zur ersten KVG-Revision im Jahr 1999 lanciert wurde, erlitt im Ständerat eine Abfuhr.

Die zweite Revision, noch unter der Leitung von Bundesrätin Ruth Dreifuss erarbeitet, wurde beinahe zu einem Erfolg. Sie sah nicht nur die Vertragsfreiheit für Fachärzte, sondern auch verschiedene Anpassungen für die Entlastung von Familien und insbesondere neue Bestimmungen im Bereich der Spitalfinanzierung vor. Ich bin der Urheber der definitiven Ablehnung dieser Revision und stolz darauf. Im Dezember 2003 stellte ich den Antrag, die Vorschläge der Einigungskonferenz abzulehnen, weil diese unter anderem auf die Einführung der Vertragsfreiheit ausgerichtet waren. Wären sie angenommen worden, hätte die FMH das Referendum ergriffen, auf das sie sich entsprechend vorbereitet hatte. Die CVP unterstützte meinen Antrag, weil nach ihrer Auffassung die Entlastung für Familien mit Kindern ungenügend war - sie wünschte eine Prämienbefreiung ab dem dritten Kind. Die SP schloss sich meinem Antrag ebenfalls an, weil mit diesem neuen Gesetz eine Liberalisierung nach dem Vor-

\section{Bislang sind wir der viel zitierten Vertragsfreiheit entronnen, die immer wieder als Wunderlösung präsentiert wird}

bild des Systems in den Vereinigten Staaten eingeführt worden wäre, ohne dass die Qualität und die Kosteneindämmung gewährleistet gewesen wären. Ausserdem war bei dieser Lösung ein Konflikt zwischen Bund und Kantonen im Planungsbereich praktisch vorprogrammiert. Der Nationalrat lehnte die Vorlage schliesslich mit 71 gegen 66 Stimmen ab. Damit konnten wir auf die Lancierung des Referendums verzichten, das die FMH bereits beschlossen hatte.
Damit hätte man eigentlich davon ausgehen können, dass die Vertragsfreiheit zumindest für eine gewisse Zeit vom Tisch war. Dies entsprach indessen nicht dem Willen von Bundesrat Pascal Couchepin, der dem Departement seit dem 1. Januar 2003 vorstand, und auch nicht der Absicht des Bundesrats. Nach einem im Schnellgang durchgeführten mündlichen Vernehmlassungsverfahren, das förmlich durchgepeitscht worden war, wurde das vom Parlament abgelehnte Gesetz erneut unterbreitet. Doch dieses Mal wurde es in mehrere getrennte Elemente aufgeteilt, um zu verhindern, dass es entweder von den Eidgenössischen Räten oder im Rahmen eines allfälligen Referendums gesamthaft verworfen werden konnte. Bislang wurden nur zwei Elemente geregelt: Das erste ist organisatorischer Art und umfasst im wesentlichen die Gesundheitskarte, und das zweite Element besteht in der Spitalfinanzierung. Die übrigen Elemente sind nach wie vor pendent. Der Ständerat wurde zum Erstrat bestimmt, um so die Dinge zu beschleunigen und rasch eine Vorlage schnüren zu können. Die kleine Kammer befasste sich im Detail mit den verschiedenen Elementen, um eine Wiederholung der katastrophalen Erfahrung vom 17. Dezember 2003 zu verhindern.

Der Ausgleichsfonds war für die Sommersession traktandiert, die Anfang Juni 2008 begann. Vorgesehen ist eine Verbesserung, die jedoch unvollständig ist, weil sie bereits die Hospitalisierung des letzten Jahres, aber keine Morbiditätskriterien umfasst. Die Vertragsfreiheit wird in dieser Herbstsession und in der nächsten Wintersession folgen. Sie hängt eng mit einer allfälligen Verlängerung des Zulassungsstopps zusammen, wobei im übrigen nicht klar ist, weshalb dies der Fall ist. Es werden weiterhin verschiedene wenig befriedigende Teillösungen unterbreitet, obwohl in letzter Zeit die Alternativpositionen der FMH an Überzeugungskraft gewonnen haben. Und gewissermassen als «Tüpfelchen auf dem i» wird mit dem Gegenvorschlag zur inzwischen zurückgezogenen SVP-Initiative «Für tiefere Krankenkassenprämien» mit der irreführenden Bezeichnung «Für Qualität und Wirtschaftlichkeit in der Krankenversicherung» der Versuch unternommen, die laufenden Debatten zu umgehen. Der Zentralvorstand, Ihre Delegiertenversammlung und Sie selbst haben ein grosses Engagement für die Abstimmungskampagne geleistet, die am 1. Juni dieses Jahres schliesslich zu einem erfreulichen Ergebnis führte. Mit 69,5\% Nein-Stimmen zum trügerischen Gegenvorschlag gab das Schweizer Volk eine klare Antwort, mit der die Vertragsfreiheit und jegliche Strategie, die auf einer Einschränkung der freien Wahl des Arztes 
durch den Patienten beruht, endgültig vom Tisch sein sollten. Trotzdem wollen gewisse Kreise diese Themen bereits wieder auf Gesetzesebene umsetzen ... und unsere Rolle im Rahmen des Gesundheitssystems positionieren. Wir haben unsere Tätigkeit auf den Grundsatz des partnerschaftlichen Dialogs, der in der gesamten Wirtschaft aner-

\section{Die Mitteilungen der FMH haben mittlerweile einen tatsächlichen Einfluss auf die Entscheide (der Kommissionen) des Stände- und des Nationalrats. Sogar der Departementsvorsteher nimmt uns mittlerweile etwas ernster}

Ich komme nun zu meinen Schlussfolgerungen. Erstens hat der Zentralvorstand in der Legislatur 2004-2008 beachtliche Fortschritte erzielt. Leider musste er sich nach den verschiedenen verwirrenden und vielfach ungünstig aufgenommenen Episoden der jüngeren Vergangenheit zuerst etwas finden. Dieser zu Beginn bestehende Mangel an einer klaren Orientierung führte zu Bedenken, die nicht immer sehr produktiv waren. Trotzdem haben sich die Kommunikation und die politischen Kontakte der FMH im Verlauf der letzten Legislatur erheblich verbessert. Ihr Präsident und verschiedene Mitglieder des Zentralvorstands nahmen regelmässig an den Sitzungen der parlamentarischen Gruppe «Gesundheit» teil. Es wurde auf unterschiedlicher Ebene interveniert: bei Parlamentarierinnen und Parlamentariern und in anderen Kreisen wie beispielsweise beim SVFB, bei anderen Verbänden und Gruppierungen und im Rahmen von persönlichen Treffen. Die Mitteilungen der FMH haben mittlerweile einen tatsächlichen Einfluss auf die Entscheide der Kommissionen des Stände- und des Nationalrats sowie der entsprechenden Räte. Sogar der Departementsvorsteher nimmt uns mittlerweile etwas ernster. Als Nationalrat fühlte ich mich nicht mehr als einsamer Akteur, der durch unangebrachte Vorstösse überrumpelt wird oder im Strudel von widersprüchlichen Aussagen versinkt. Im Gegenteil, meine Vorstösse profitierten von einer immer besser strukturierten Unterstützung. Dies führte zur Einrichtung einer FMHKommunikationsabteilung, die diesen Namen verdient und die Unterstützung einer spezialisierten Agentur in Anspruch nehmen kann.

Zweitens kann die FMH nur politische Erfolge erzielen, wenn wir Vorschläge unterbreiten, die von öffentlichem Interesse sind. Wenn wir uns ausschliesslich auf unsere unmittelbaren Standesinteressen konzentrieren, sind unsere Erfolgsaussichten gleich null. Es ist daher von ausschlaggebender Bedeutung, dass wir unsere Tätigkeit kannt ist, sowie auf die Gewährleistung der therapeutischen Freiheit fokussiert. Es besteht zunehmend die Tendenz, dass das Behandlungsverfahren direkt im Gesetz verankert wird - dies ist insbesondere beim Betäubungsmittelgesetz und bei der Transplantationsverordnung der Fall. Angesichts der zahlreichen Gesetze, die gegenwärtig erarbeitet werden oder einer Revision bedürfen Fortpflanzungsmedizingesetz, Bundesgesetz über die Forschung am Menschen, Präventionsgesetz und insbesondere das KVG usw. -, ist die Versuchung gross, Vorschriften zu erlassen oder das System einem leitenden Organ oder ganz einfach den Krankenkassen zu unterstellen. Der einzige glaubwürdige Ansatz besteht darin, dass man einfache und klare allgemeine Interessengrundsätze geltend macht. Unsere Arbeitsbedingungen sind Teil von anderen Verhandlungen, deren Ausgang von Grundsatzerfolgen abhängt, die wir in anderen Bereichen erzielen. Es ist daher völlig illusorisch und kontraproduktiv, wenn man das Boot mit einer Vielzahl von weiteren Überlegungen überladen will. Diese mögen zwar nach unserer Auffassung von Bedeutung sein, doch sie führen nur zu Verwirrung und zu Argwohn hinsichtlich unserer tatsächlichen Zielsetzungen.

Drittens hängt eine der grundlegenden Strategien zur Erzielung dieser Erfolge von der Einheit $a b$, die unsere Standesorganisation unter Beweis stellt. Es ist daher sehr wichtig, dass einheitlich kommuniziert wird. Nichts ist schädlicher als das parallele Vorgehen einzelner Interessengruppen. Mit der neu geschaffenen Delegiertenversammlung haben wir nun Strukturreformen eingeleitet. Die Delegiertenversammlung ermöglicht die Kommunikation und muss den Aufbau ausreichender Vertrauensbeziehungen erlauben. Das Wort «Vertrauen» ist in diesem Zusammenhang von absolut zentraler Bedeutung. Im Rahmen von SwissDRG wurden verschiedene Arbeitsgruppen gebildet, um den Informationsfluss und regelmässige Interventionen der Fachgesellschaften 
und der Fachärzte im Zusammenhang mit diesen Problemen zu gewährleisten. Unser Delegierter im Verwaltungsrat, Dr. P.-F. Cuénoud, erstattet der Delegiertenversammlung regelmässig Bericht. Dies ist ein gutes Beispiel für einen Ansatz, der sowohl auf interner als auch auf externer Ebene zu erfreulichen Ergebnissen führt. Dem Präsidenten und seinen Vizepräsidenten muss das gleiche Vertrauen entgegengebracht werden. Es muss ihnen auch die Kompetenz eingeräumt werden, abhängig von den Ereignissen dringende Massnahmen zu ergreifen. Diese müssen innerhalb der allgemeinen Logik realisiert werden, die den verschiedenen zuständigen Stellen bereits dargelegt wurde.

Schliesslich muss unsere politische Kultur auf allen Ebenen verbessert werden. Die Ärztinnen und Ärzte können sich nicht mehr darauf beschränken, ganz einfach entschlossen Forderungen zu stellen, um ihre Ziele durchzusetzen. Die Zeiten haben sich geändert. Die Ärztinnen und Ärzte sind heutzutage eine Interessen- und Einflussgruppe wie jede andere. Es ist daher wichtig, dass man sich als erstes umfassend dokumentiert und gewisse Regeln berücksichtigt. Die erste Regel ist die Respektierung des Gegners. Die zweite Regel besteht im Verfassen von kurzen und einfachen Mitteilungen, die rasch verständlich sind. Parlamentarierinnen und Parlamentarier lesen kaum mehr als eine A4-Seite. Die dritte Regel besteht darin, dass demokratische Entscheide zu respektieren sind und dass unsere Institutionen einheitlich kommunizieren müssen. Ohne diese Anstrengungen können keine Erfolge erzielt werden.

Die FMH hat sich tiefgreifend reorganisiert, und es wurden demokratischere Strukturen geschaffen. Ihre politische Glaubwürdigkeit ist in den letzten Jahren deutlich gestiegen. Auf dieser Grundlage können wir mehr Erfolge erzielen als in der Vergangenheit. Der neue Ansatz muss mit einem partnerschaftlichen Dialog und mit einer konstruktiven Einstellung einhergehen. Es bleibt zu hoffen, dass diese beiden Elemente noch verbessert werden. Im Gesetz sollte dieser Ansatz als oberster Grundsatz für die Suche nach Lösungen verankert werden. Ich wünsche dem neuen Zentralvorstand und der FMH alles Gute und bedanke mich für das Vertrauen, das mir in all diesen Jahren entgegengebracht wurde. 